\title{
Split of an Optimization Variable in Game Theory
}

\author{
R. Aboulaich ${ }^{1 *}$, A. Habbal ${ }^{2}$ and N. Moussaid ${ }^{1}$ \\ ${ }^{1}$ LERMA, E.M.I., Avenue Ibn Sina B.P 765, Agdal, Rabat. Morocco \\ ${ }^{2}$ LJAD, University of Nice Sophia-Antipolis, Valrose, 06108 Nice Cedex 2, France
}

\begin{abstract}
In the present paper, a general multiobjective optimization problem is stated as a Nash game. In the nonrestrictive case of two objectives, we address the problem of the splitting of the design variable between the two players. The so-called territory splitting problem is solved by means of an allocative approach. We propose two algorithms in order to find fair allocation tables.
\end{abstract}

Key words: multiobjective optimization, concurrent optimization, split of territories, Nash equilibrium, Pareto front

AMS subject classification: $91 \mathrm{~A} 10$

\section{Introduction}

Let us consider the multiobjective program defined by

$$
(M) \min _{x}\left\{\begin{array}{l}
f_{1}(x) \\
f_{2}(x)
\end{array}\right\},
$$

when the cost functions are concurrent, the problem (M) must be reformulated to make sense. To this end, a classical approach used to tackle multiobjective optimization is to define a scalar anzats, generally a convex combination of the multiple criteria. This approach introduces however an arbitrary choice of weights. In the convex case, taking all possible optima obtained for all possible weights yields the Pareto front, which is of high importance to understand the tradeoff between competitive criteria. Unfortunately, the determination of the Pareto front is generally very expensive. So, it could be of interest to reframe the multiobjective optimization problem as a Nash game $[2,3]$ : we split the original design variable into two strategies, formally we denote

*Corresponding author. E-mail: aboulaich@emi.ac.ma 
$x=(U, V)$. Then we look for a Nash equilibrium, defined as the couple solution to

$$
(P)\left\{\begin{array}{l}
\min _{U} f_{1}(U, V), \\
\min _{V} f_{2}(U, V) .
\end{array}\right.
$$

The main difficulty of the theoretic-game approach is the determination of the best, non arbitrary, partition of the variable $x$ into the artificial variables $U$ and $V$. This question, the main concern of our paper, is a nontrivial and difficult problem. To our knowledge, there are very few contributions to the study of this territory splitting problem. In [4] Désidéri proposes an algorithm of territory splitting using the eigenvectors of the Hessian matrix of one criteria $f_{1}$, which somehow plays the role of a preferred objective. In the following we propose two algorithms in order to find a Nash equilibrium using a splitting of the strategy spaces when no cost is preferred among others.

\section{Splitting algorithms}

Pure allocation tables are any elements $P$ and $Q$ from $\{0,1\}^{n}$ that satisfy $P_{i}+Q_{i}=1$ for $1 \leq$ $i \leq n$. Mixed allocations are obtained by convexification of the set of pure ones. We also drop the mutual exclusivity constraint, to allow both players to share the same variable. To split the optimization variable, we construct a sequence of two tables of allocation $P^{(m)}$ and $Q^{(m)}$ in $[0,1]^{n}$, and we use an auxiliary function $f$ defined by (2.2) from $f_{1}$ and $f_{2}$. In order to construct the sequences $P^{(m)}$ and $Q^{(m)}$, and to determine the Nash equilibrium $x_{N E}$, we propose the two following algorithms.

\subsection{Algorithm 1 (AG1), heuristic allocation tables}

Algorithm 1. Step 1: Set $m=0$. Starting from any initial guess $x^{(0)}, y^{(0)}$ in $\mathbb{R}^{n}$, and $\rho>0$ is a constat descent step, compute $P^{(0)}$ and $Q^{(0)}$ by:

$$
\left\{\begin{array}{r}
\min _{x \in \mathbb{R}^{n}} f_{1}(x), \quad x^{(k+1)}=x^{(k)}-\rho \nabla f_{1}\left(x^{(k)}\right), \quad k \geq 0, \\
P_{j}^{(0)}=\frac{\Sigma_{k}\left|x_{j}^{(k+1)}-x_{j}^{(k)}\right|}{\Sigma_{k}\left\|x^{(k+1)}-x^{(k)}\right\|}, \\
\min _{y \in \mathbb{R}^{n}} f_{2}(y), \quad y^{(k+1)}=y^{(k)}-\rho \nabla f_{2}\left(y^{(k)}\right), \quad k \geq 0, \\
Q_{j}^{(0)}=\frac{\Sigma_{k}\left|y_{j}^{(k+1)}-y_{j}^{(k)}\right|}{\Sigma_{k}\left\|y^{(k+1)}-y^{(k)}\right\|},
\end{array}\right.
$$

then initially set.

$$
x_{N E}^{(0)}=P^{(0)} \cdot x^{*}+Q^{(0)} \cdot y^{*},
$$

where $x^{*}$ is the solution to $\min _{x} f_{1}(x)$ and $y^{*}$ the solution to $\min _{x} f_{2}(x)$, the dot denotes the Hadamard product. We now define the function $f$ by

$$
f(x)=f_{1}(\bar{x})+f_{2}(\bar{y})
$$


where $\bar{x}=P^{(m)} \cdot x+Q^{(m)} \cdot x_{N E}^{(m)}$ and $\bar{y}=P^{(m)} \cdot x_{N E}^{(m)}+Q^{(m)} \cdot x$.

Step 2: Compute $x_{\text {opt }}^{(m)}$ the solution to $\min _{x} f(x)$ and update $P^{(m+1)}$ and $Q^{(m+1)}$ as follows

$$
\left\{\begin{array}{c}
\min _{x} f(x), \quad x^{(k+1)}=x^{(k)}-\rho \nabla f\left(x^{(k)}\right), \quad k \geq 0, \\
P_{j}^{(m+1)}=\frac{\sum_{k}\left|\left(\nabla f_{1}\left(P^{(m)} \cdot x^{(k+1)}+Q^{(m)} \cdot x_{N E}^{(m)}\right)\right)_{j}\right|}{\Sigma_{k}\left\|\nabla f_{1}\left(P^{(m)} \cdot x^{(k+1)}+Q^{(m)} \cdot x_{N E}^{(m)}\right)\right\|}, \\
Q_{j}^{(m+1)}=\frac{\sum_{k}\left|\left(\nabla f_{2}\left(Q^{(m)} \cdot x^{(k+1)}+P^{(m)} \cdot x_{N E}^{(m)}\right)\right)_{j}\right|}{\sum_{k}\left\|\nabla f_{2}\left(Q^{(m)} \cdot x^{(k+1)}+P^{(m)} \cdot x_{N E}^{(m)}\right)\right\|}, \\
x_{N E}^{(m+1)}=P^{(m)} \cdot x_{o p t}^{(m)}+Q^{(m)} \cdot x_{N E}^{(m)},
\end{array}\right.
$$

while $\quad\left\|x_{N E}^{(m+1)}-x_{N E}^{(m)}\right\|>$ test, set $m=m+1$, redo step 2.

\subsection{Algorithm 2 (AG2), optimized allocation tables}

Algorithm 2. 1. initial step 1 as in (AG1),

2. given $P^{(m)}$ and $Q^{(m)}$, compute $x_{\text {opt }}^{(m)}$ the solution to $\min _{x} f(x)$,

3. solve the minimization problem $\min _{P} f\left(P . x_{o p t}^{(m)}+Q^{(m)} \cdot x_{N E}^{(m)}\right)$ to get $P^{(m+1)}$,

4. solve the minimization problem $\min _{Q} f\left(P^{(m)} \cdot x_{N E}^{(m)}+Q . x_{o p t}^{(m)}\right)$ to get $Q^{(m+1)}$,

5. $\operatorname{set} x_{N E}^{(m+1)}=P^{(m+1)} \cdot x_{o p t}^{(m)}+Q^{(m+1)} \cdot x_{N E}^{(m)}$,

6. while $\left\|x_{N E}^{(m+1)}-x_{N E}^{(m)}\right\|>$ test, set $m=m+1$, redo 2 .

We pay a particular attention to check if the Nash equilibria computed by the proposed algorithms belong to the Pareto front, also known as the set of non-dominated strategies (the meaning is obvious from the definition of the front) [1]. We set, $f_{\lambda}=\lambda f_{1}+(1-\lambda) f_{2}, \lambda \in[0,1]$. For each $\lambda$ we compute the optima, the set of which forms the Pareto front (at least in the convex case). Below we present some numerical results obtained by algorithms 1 and 2 .

\section{Numerical results}

We consider a simple illustrating case where $f_{1}(x)=\|A x-b\|^{2}$ for the first player, and $f_{2}(x)=$ $\|C x-d\|^{2}$ for the second one, where $A$ and $C$ are two $n \times n$ matrices, and $b$ and $d$ are vectors, that is $n \times 1$ matrices. We observe that the two algorithms converge. In example 1 , see figure 1 and figure 2, we obtain the convergence to a Nash equilibrium which lies on the Pareto front, and corresponds to the optimum of $f_{\lambda}$ for a value $\lambda=1 / 2$. In example 2 , see figure 3 and figure 4 , the 
best computed Nash equilibrium is close to the Pareto front but not on it. Moreover, it corresponds to a strategy that is more advantageous for the cost $f_{2}$.

Example 1. $A=C=I d, b=[1,-2,2,9,1,2,9], d=[5,1,3,-8,-6,0,4]$
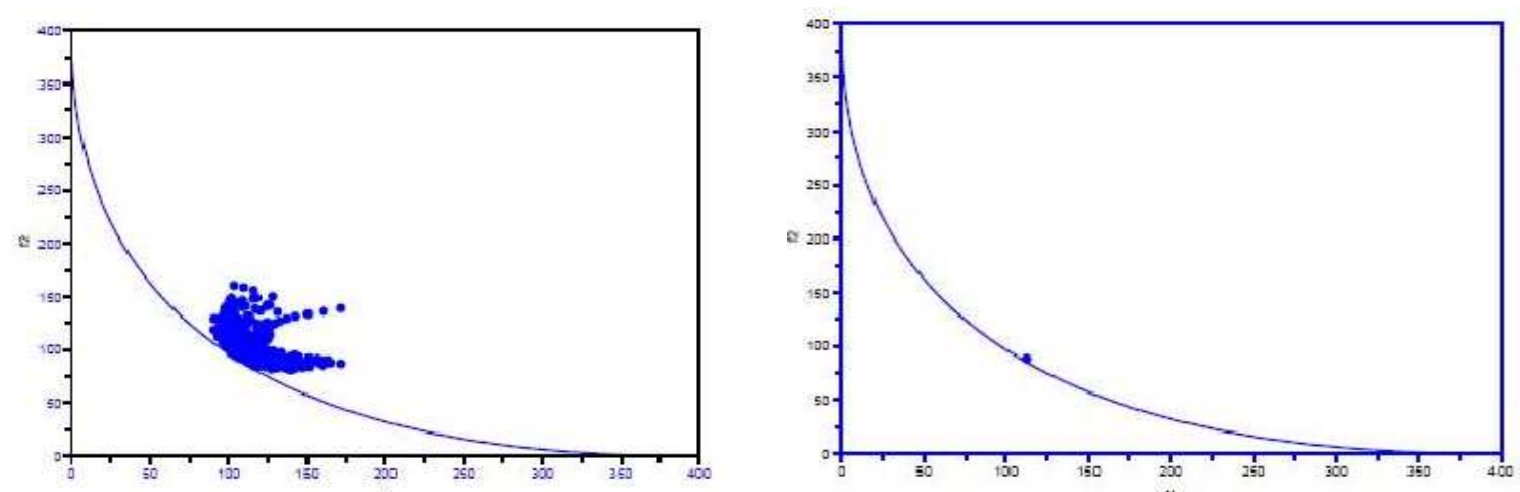

Figure 1: Pareto front and Nash equilibriums (AG1). Pareto front and the last Nash equilibrium found by (AG1)
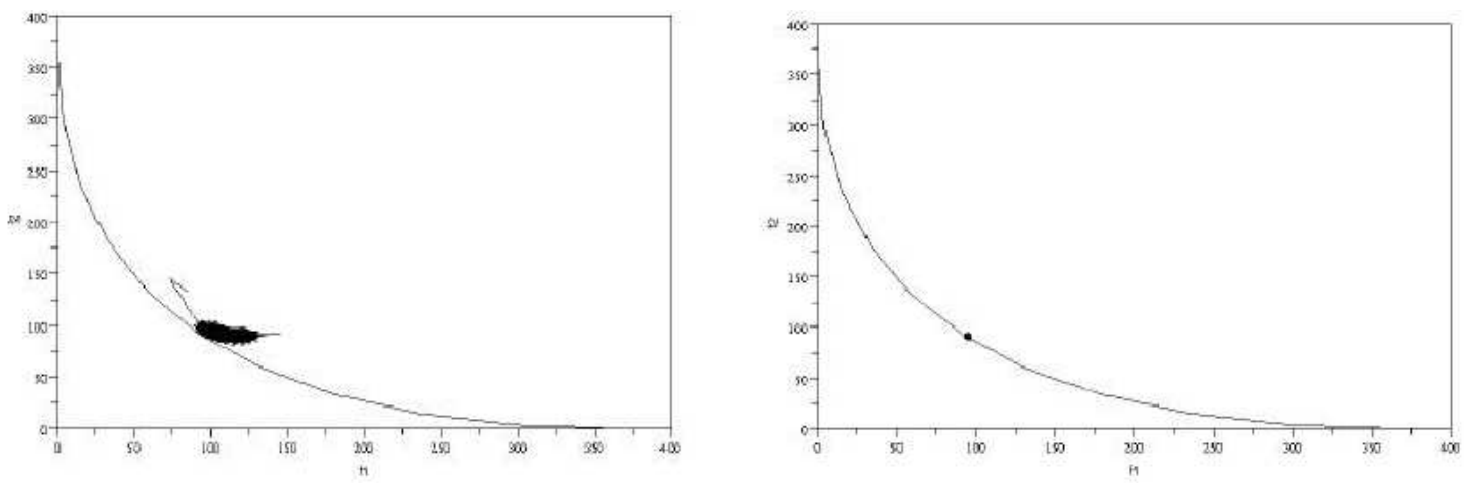

Figure 2: Pareto front and Nash equilibriums (AG2). Pareto front and the last Nash equilibrium found by (AG2)

Example 2. $A=\operatorname{tridiag}[1,-2,1], C=A ; b=\operatorname{rand}(n, 1), d=10 * b, n=50$ 

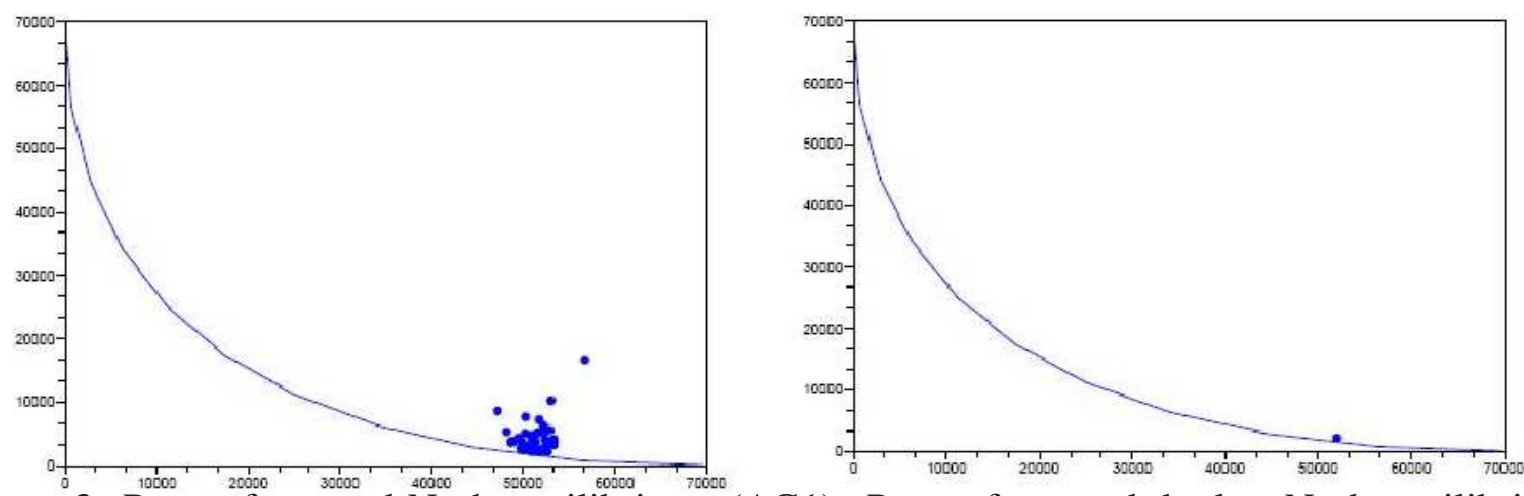

Figure 3: Pareto front and Nash equilibriums (AG1). Pareto front and the last Nash equilibrium

found by (AG1)
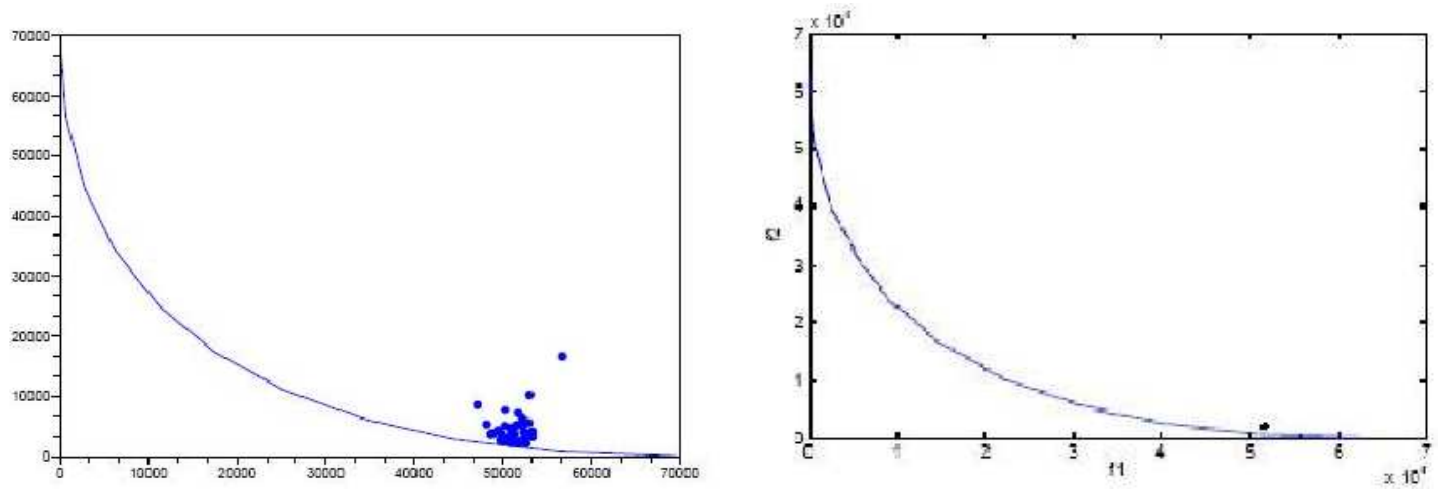

Figure 4: Pareto front and Nash equilibriums (AG2). Pareto front and the last Nash equilibrium found by $(\mathrm{AG} 2)$

\section{Conclusion}

Both algorithms yield successive iterations that lie close to if not on the Pareto front. Ongoing work is in progress to address weak points such as the computational cost that must be lowered or the heuristic determination of the allocation tables that must be at least statistically driven.

\section{Acknowledgements}

This work was supported by Action Integrée Franco-Morocaine MA/05/115 and the SARIMA program. 


\section{References}

[1] J. P. Aubin. Mathematical methods of game and economic theory. North-Holland Publishing Co. Amsterdam, New York, 1979.

[2] A. Habbal. A topology Nash game for tumoral antangiogenesis. Struct. Multidisc. Optim., 30 (2005), 404-412.

[3] A. Habbal, J. Petersson, M. Thellner. Multidisciplinary topology optimization solved as a Nash game. Struct. Multidisc. Optim., 61 (2004), 949-963.

[4] J. A. Désidéri. Split of territories in concurrent optimization. Rapport de recherche, INRIA, 2007. 